\title{
Insights into the Fungal Community and Functional Roles of Pepper Rhizosphere Soil under Plastic Shed Cultivation
}

\author{
Shi Yao ${ }^{1,2,+}$, Xiaona $\mathrm{Li}^{1,2,+}$, Hu Cheng ${ }^{3}$, Kaining Sun ${ }^{4}$, Xin Jiang ${ }^{1,2}$ and Yang Song ${ }^{1,2, *}$ \\ 1 CAS Key Laboratory of Soil Environment and Pollution Remediation, Institute of Soil Science, \\ Chinese Academy of Sciences, Nanjing 210008, China; yaoshi@issas.ac.cn (S.Y.); xnli@issas.ac.cn (X.L.); \\ jiangxin@issas.ac.cn (X.J.) \\ 2 University of Chinese Academy of Sciences, Beijing 100049, China \\ 3 College of Biology and the Environment, Nanjing Forestry University, Nanjing 210037, China; \\ hucheng@njfu.edu.cn \\ 4 Institute of Vegetables and Flowers, Shandong Academy of Agricultural Sciences, Jinan 250100, China; \\ sunkaining-123@163.com \\ * Correspondence: ysong@issas.ac.cn; Tel.: +86-258-688-1193 \\ + These two authors contributed equally to this work.
}

Received: 30 October 2020; Accepted: 13 November 2020; Published: 17 November 2020

\begin{abstract}
The rhizosphere fungal community is essential for determining plant health and improving crop productivity. The fungal community structure and functional roles in the plastic shed soils were explored using high throughput sequencing and FUNGuild in this study. The fungal community structures shifted between the rhizosphere and non-rhizosphere soils. The greatest abundance variation was observed for the rare fungal members with relative abundances $<0.1 \%$. In the rhizosphere soil of pepper, the abundance of the genera Purpureocillium, Metacorgyceps, Arthrobotrys, Cephalotheca, and Scedosporium increased significantly, among which, Purpureocillium, Arthrobotrys and Metacorgyceps exhibited biocontrol characteristics. Co-occurrence network analysis revealed different interactions of fungal communities in the rhizosphere and non-rhizosphere soils, both of which were dominated by low abundance members. More positive correlation was identified among the rare members, the fungal pathotroph functions and phthalate acid ester in the rhizosphere soil. This study highlights the important niche of the rare fungal members in soil microbial ecology under plastic shed cultivation.
\end{abstract}

Keywords: co-occurrence network; fungal community; fungal functional role; plastic shed cultivation; rhizosphere

\section{Introduction}

Plastic shed cultivation was developed in response to the increasing global population and food demand. The area occupied by global plastic shed cultivation has increased 4.3 times in the past two decades [1]. Furthermore, the total area of plastic shed cultivation in China accounts for more than $80 \%$ of that in the world [2]. Pepper is a main plastic shed cultivated vegetable in China with 1.33 million $\mathrm{hm}^{2}$ of planting area, yielding 28 million tons annually [3,4]. Plastic shed cultivation supplies a great number of off-season vegetables for human consumption. However, the extensive use of plastic films results in phthalate acid ester (PAE) contamination in plastic shed soils [5]. Additionally, the large agricultural input, high soil temperature and airtight environment associated with plastic shed cultivation usually leads to decreased soil quality [6] and microbial diversity [5]. Soil microbiomes 
exert a decisive role during carbon and nitrogen cycling; terrestrial ecosystem decomposition; and other multifunctional aspects [7].

Fungal community is an indispensable component of microbiomes and it plays an important role in mediating the terrestrial ecology, such as nitrogen and carbon cycling, parasitism, and pathogenicity $[7,8]$. The fungal community could impact plant growth by interacting with pathogens and plants [9]. The shift of fungal community structure could be affected by soil properties and cultivation patterns [10]. During plastic shed cultivation, the special planting environment has a significant impact on the fungal community structure and could even inhibit the growth of fungi, thereby disturbing the ecological balance of the microorganisms [11]. It has been reported that when compared to open soil cultivation, the microbial biomass decreases and the fungal community degenerates in plastic shed cultivated soil [11]. Moreover, our previous research, which was based on a twenty-year cultivation study, confirmed that the plastic shed cultivation mode reduces fungal richness and diversity [5]. Meanwhile, it remains unclear how the fungal community's functional roles shift during plastic shed cultivation, especially within the plant rhizosphere.

The plant rhizosphere is the most sensitive hotspot location in which microbiomes respond to their surroundings in soils [12]. The interaction between the soil microbiome and plant roots dominates the rhizosphere process. Plastic shed cultivation commonly causes stunted plant growth and heavy pest infestation, which are closely related to the plant rhizosphere [13,14]. For example, peanut root exudates greatly impact the fungal community, leading to an increase in relative abundance of soil-borne pathogens, particularly F. oxysporum [15]. Furthermore, certain root exudates, such as cinnamic, myristic and fumaric acids, have been shown to increase the presence of fungal pathogens in plant rhizosphere soil [16]. However, the influence of the pepper rhizosphere condition on shaping the fungal community in plastic shed soil has been rarely reported [17].

The combination of microbial community structure and functional roles are at the core of understanding the role of microorganisms within an ecosystem [18]. Recently, the python-based FUNGuild tool was used to identify specific ecological categories of fungi through its simple and convenient data processing [19]. Feng et al. reported that some fungi play an important role in restoring the stability of aggregates with FUNGuild [20]. Other studies have used FUNGuild to elucidate the specific roles played by different fungal communities in rhizosphere soils and compost [21,22]. To our knowledge, there is a lack of reports on the fungal functional roles in the pepper rhizosphere and non-rhizosphere soil under plastic shed cultivation. Therefore, clarifying specific shifts in the fungal community structure and functional roles in plastic shed pepper fields will provide new insight for the management and sustainable agricultural production of plastic shed cultivation [23].

This study aimed to clarify the shifts in the soil fungal community structure and functional roles of the pepper rhizosphere in response to the plastic shed cultivation conditions. The rhizosphere and non-rhizosphere soil samples were collected from a long-term plastic shed vegetable cultivation site. The soil fungal community structures were analyzed by sequencing and then the potential fungal functional roles were predicted using FUNGuild. This study will provide helpful information for the management of the soil microbial ecology during plastic shed cultivation.

\section{Materials and Methods}

\subsection{Soil Sample Collection}

The plastic shed soil site, with the soil type of fulvo-aquic soil, was located in Weifang, Shandong province, China. After 4 months of pepper cultivation, which is the maturity period of pepper, all the soils were sampled at a depth of $20 \mathrm{~cm}$ from a single greenhouse with an area greater than $2000 \mathrm{~m}^{2}$. The fertilizer was applied in combination with fertilizer and chicken manure, respectively $1500 \mathrm{~kg} / \mathrm{ha}$ $(\mathrm{N} / \mathrm{P} / \mathrm{K}, 15: 15: 15)$ and $7500 \mathrm{~kg} / \mathrm{ha}$ [5]. The $5 \mathrm{~mm}$ of soil attached to the pepper roots were taken as rhizosphere soil. Then, the corresponding non-rhizosphere soil that was $1 \mathrm{~m}$ away from the pepper roots was also sampled. Five replicates were sampled to ensure the reliability of the field experiment 
data [2] and then all samples were carried to the lab in cool boxes. Subsamples for DNA extraction were stored at $-80{ }^{\circ} \mathrm{C}$. The remaining subsamples were used for soil property analysis after being air-dried and then sieved to soil particle size less than $2 \mathrm{~mm}$. The soil properties were determined using the reference standard determination method, while PAE extraction and detection in the soil was conducted according to [5].

\subsection{DNA Extraction and High-Throughput Sequencing}

The E.Z.N.A. ${ }^{\circledR}$ Soil DNA Kit (Omega Bio-tek, Norcross, GA, USA) was used to extract microbial DNA from $0.5 \mathrm{~g}$ of soil. The DNA extraction procedure was as follows: First, the soil sample was mixed with glass beads $(0.5 \mathrm{~g})$, SLX-Mlus Buffer $(1 \mathrm{~mL})$ and DS Buffer $(0.1 \mathrm{~mL})$ in a $15 \mathrm{~mL}$ centrifuge tube. The samples were vortexed to lyse the cells; and then the tubes were incubated at $70{ }^{\circ} \mathrm{C}$ for $10 \mathrm{~min}$ with a slight shaking. Next, the tubes were centrifuged at $3000 \mathrm{rpm}$ for $3 \mathrm{~min}$ and then the supernatant with the volume of $0.8 \mathrm{~mL}$ was mixed with $0.27 \mathrm{~mL}$ of P2 Buffer in a $2 \mathrm{~mL}$ centrifuge tube and incubated for 5 min under cold conditions. After a subsequent centrifugation, the DNA supernatant was transferred into a new $2 \mathrm{~mL}$ centrifuge tube. Next, isopropanol and Elution Buffer were used successively, followed by a $2 \mathrm{~min}$ incubation with $0.1 \mathrm{~mL}$ of HTR Reagent. A HiBind ${ }^{\circledR}$ DNA Mini Column was used for DNA purification and then the DNA sample finally dissolved in $0.1 \mathrm{~mL}$ of Elution Buffer. The DNA samples were stored at $-20^{\circ} \mathrm{C}$ until downstream analyses. The Nano drop system (Thermo Scientific) was used to determine the purity and concentration of DNA.

The fungi-specific primers ITS1F (-CTTGGTCATTTAGAGGAAGTAA-) and ITS2R (-GCTGCGTTCTTCATCGATGC-) were used for polymerase chain reaction (PCR). The PCRs $(20 \mu \mathrm{L})$ were performed with DNA sample $(10 \mathrm{ng})$, FastPfu Buffer $(4 \mu \mathrm{L}), 2.5 \mathrm{mM}$ dNTPs $(2 \mu \mathrm{L})$, each primer $(0.8 \mu \mathrm{L})$, FastPfu Polymerase $(0.4 \mu \mathrm{L})$, BSA $(0.2 \mu \mathrm{L})$ and double-distilled $\mathrm{H}_{2} \mathrm{O}(0.2 \mu \mathrm{L})$. Then the PCR was amplified according to the program described by [5]. The amplicons were analyzed by $2 \%$ agarose gel. Triplicate amplification was used for each sample and then pooled for sequencing. Amplicon sequencing was carried out on Illumina MiSeq platform (Illumina, San Diego, CA, USA). After sequencing, the raw sequences were analyzed with the QIIME 1.9.0-dev pipeline and FLASH. The data filtering was completed by removing bases with a quality score less than 20 and splice contaminated sequences to obtain high-quality target sequences for subsequent analysis. Operational taxonomic units (OTUs) were classified from the sequences at the similarity of $97 \%$ with the USEARCH algorithm (http://drive5/uparse/). Accession number of the raw reads submitted to the Sequence Read Archive database of National Center for Biotechnology Information is SRP182013.

\subsection{FUNGuild Analysis}

Fungal ecological function prediction and categorization was performed via the FUNGuild v 1.0 database. At the time of analysis, the database contained 9476 entries, of which $66 \%$ were at the genus level and 34\% were at the species level. The guilds' information was accepted only if the identity and coverage were more than $97 \%$. The evaluation results were then ranked as "highly probable", "probable", or "possible" after comparison in the fungal database, while unmatched taxa were named "unassigned" and subsequently excluded. To interpret the data in an ecological context, the selected OTUs were classified according to the main feeding habits of fungi. Finally, 240 OTUs were assigned to 8 trophic modes as follows: saprotroph, 118 OTUs; symbiotroph, 2 OTUs; pathotroph, 28 OTUs; saprotroph-symbiotroph, 19 OTUs; pathotroph-saprotroph, 7 OTUs; pathotroph-symbiotroph, 4 OTUs; pathotroph-saprotroph-symbiotroph, 47 OTUs and pathogen-saprotroph-symbiotroph, 15 OTUs.

\subsection{Data Analysis and Statistics}

The diversity of the fungal communities was expressed by the Chao and Shannon indices with Mothur v.1.30.1. Then the significant difference post hoc comparison was tested at the $p<0.05$ level by one-way analysis of variance in Statistical Product and Service Solutions V20.0. Venn plots of fungal communities and functional roles were created using the "venerable" package in R 3.4.0. 
Significant fungal biomarkers were detected using linear discriminant analysis (LDA) effect size (LEfSe), basing on the assigned fungal taxa at LDA > 3.0. The network of soil fungal community members, functional roles and soil properties was visualized using Gephi 0.9.2. The Spearman correlation index $(>0.6)$ and $p$ values $(<0.05)$ were calculated using the "psych" package in R 3.4.0.

\section{Results and Discussion}

\subsection{Shifts in Fungal Community Structure in Soil}

A total of 381 fungal OTUs were obtained from sequencing, of which 64 unique OTUs were detected in the rhizosphere and 62 unique OTUs were detected in non-rhizosphere soils, indicating that the plant rhizosphere can drive a unique soil microbial community [24,25]. In addition, both the fungal diversity and richness were higher in the rhizosphere soil than those in the non-rhizosphere soil in the presence of plastic shed condition (Figure 1a).

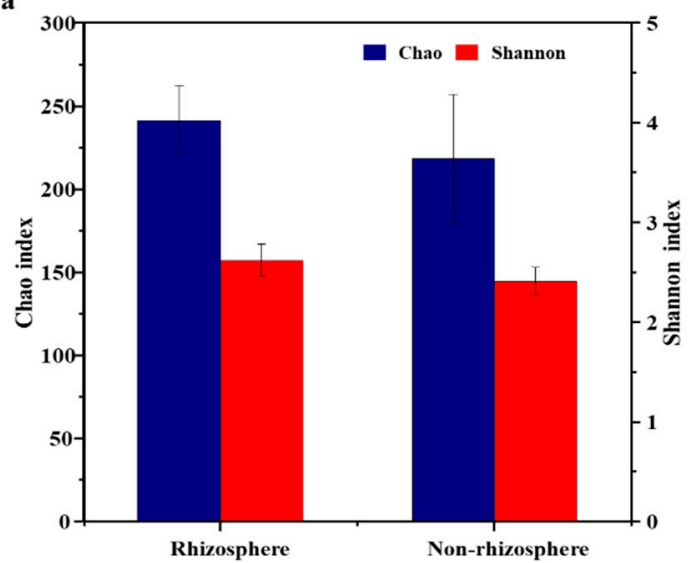

b

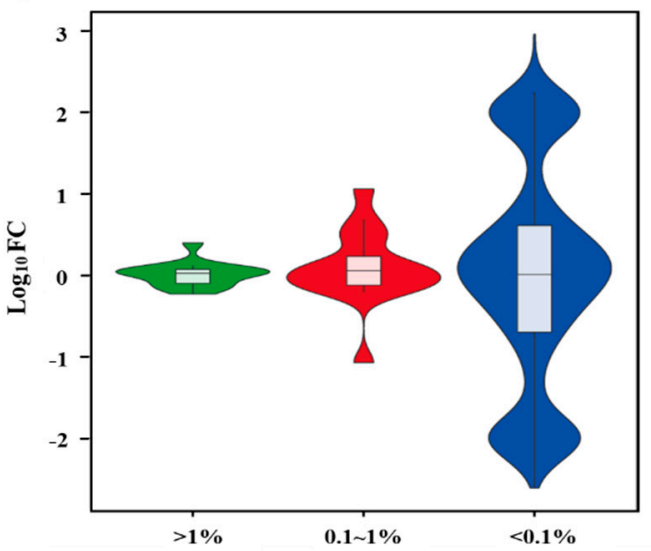

Figure 1. Chao and Shannon indices of the fungal communities in the soils (a). The effect of different soil environments (rhizosphere and non-rhizosphere) on the fungal community shift is shown by violin plots (b). The relative abundance of the operational taxonomic units (OTUs) are categorized as high-abundance $(>1 \%)$, medium-abundance $(0.1 \sim 1 \%)$ or low-abundance $(<0.1 \%)$. FC $=$ the abundance of OTUs in rhizosphere soils/the abundance of OTUs in non-rhizosphere soils.

Ascomycetes and Zygomycetes were the two main fungal phyla, accounting for $93.2 \%$ and $5.3 \%$ of the whole community, respectively (Figure 2a). Through the LEfSe analysis, some fungal community members exhibited a significant shift between the rhizosphere and non-rhizosphere soils. At the genus level, the abundance of Purpureocillium, Metacorgyceps, Arthrobotrys, Cephalotheca, Scedosporium and classified-Lasiosphaeriaceae significantly increased in the rhizosphere environment, while the abundances of Pseudaleuria and classified-Hypocreales were significantly lower in the pepper rhizosphere than those in the non-rhizosphere soil under the plastic shed condition (Figure 2b). Specifically, Purpureocillium, an entomopathogenic fungus within a family of soil-borne micromycetes, increased the most in the pepper rhizosphere soil. This genus was also reported to be beneficial for the defense from nematodes and nutrient uptake by habanero chili pepper (Capsicum Chinese Jacq.), thereby facilitating plant growth $[26,27]$. Meanwhile, other fungal genera, such as Arthrobotrys and Metacordyceps, reportedly play important roles in defending plants against nematode infestations [25,28], which can damage plant growth and result in lower yields, especially for pepper cultivation [29]. Antagonistic microorganisms that act against nematodes have also been reported in soils with a combination of various planting patterns [30]. Therefore, this result indicates that pepper roots could recruit nematode-resistant fungi in the rhizosphere soil during plastic shed cultivation, thus benefiting its growth. This could be one of the most important ecological functions of fungi within the plant rhizosphere soil, especially for soils subjected to the plastic shed agricultural practice. Additionally, the pepper rhizosphere significantly 
enriched Scedosporium. This may be because its presence is associated with the phosphorus content of the soil, particularly in soils with a moderate and high phosphorus content [31]. In this study, the phosphorus content in the rhizosphere environment soil was indeed greater than that in the non-rhizosphere soil [32]. Meanwhile, the abundance of Pseudaleuria was significantly decreased in the rhizosphere soil (Figure 2b). It was reported that manure application increases the abundance of Pseudaleuria [33]. Thus, the low abundance of Pseudaleuria in the rhizosphere soil of this study may be explained because the turnover of organic fertilizers in the pepper rhizosphere was faster than that in the bulk soil, thereby changing the ideal conditions for Pseudaleuria.

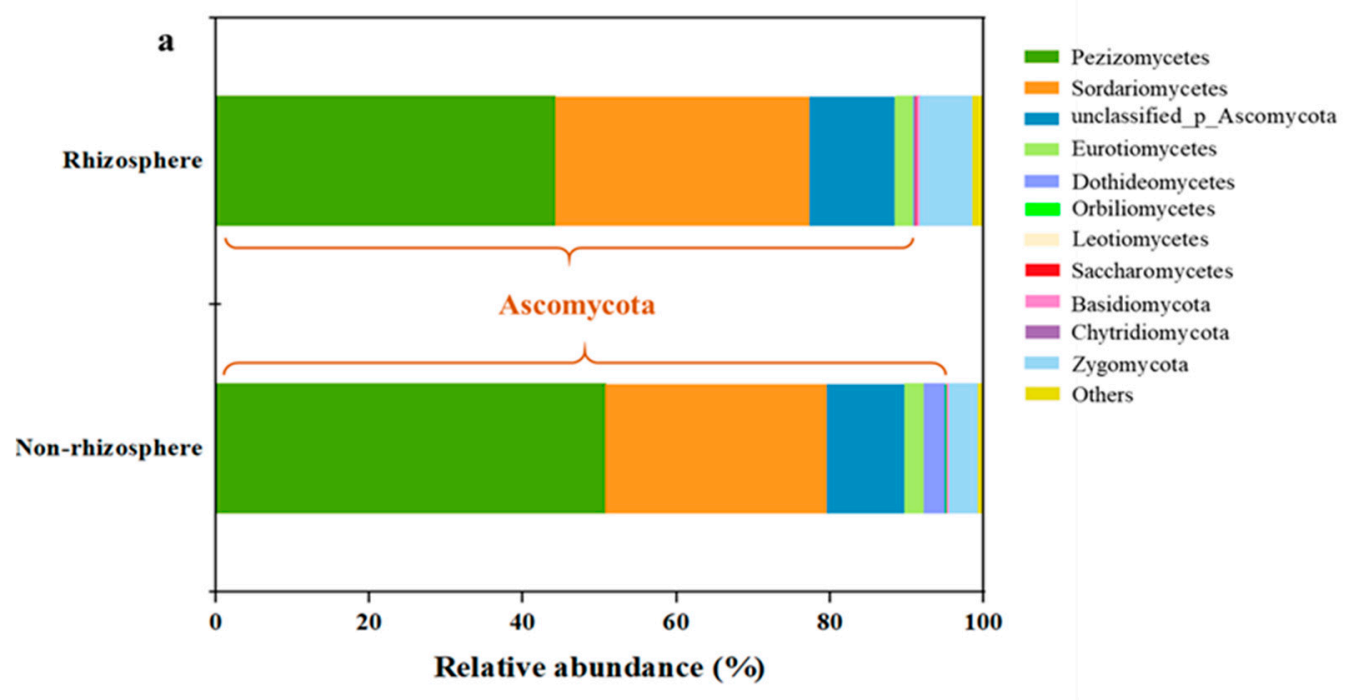

b

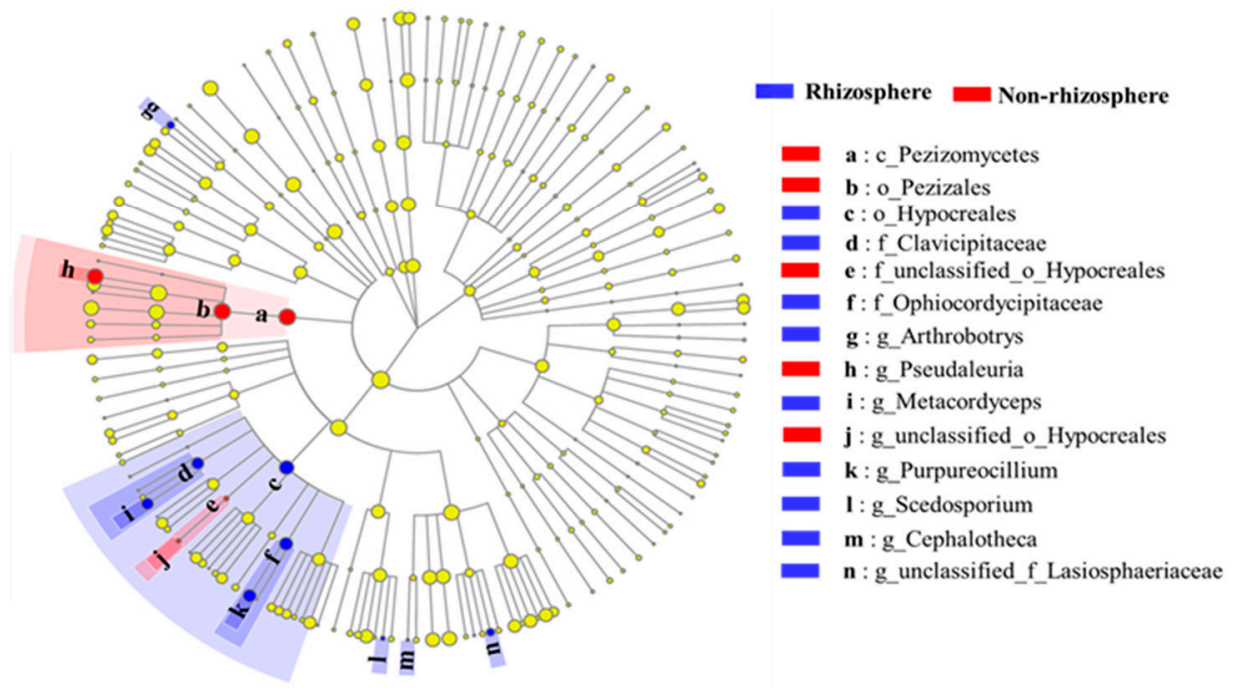

Figure 2. The abundance and composition of the fungal community at the phylum/class levels in soils (a). The response of the significantly fungal members from phyla to genera in the rhizosphere (blue) and non- rhizosphere soils (red) (b).

\subsection{Interaction of Fungal Communities}

Determining the abundance distribution of fungi is of great help for understanding the ecological processes of microorganisms [34]. Thus, the effect of the rhizosphere and non-rhizosphere environments on the variation of fungal abundance was determined (Figure $1 b$ ). The $\log _{10}$ (FC) $>0$ indicates that the abundance of fungi increased in the rhizosphere environment, and vice versa (detailed calculation is shown in Figure 1). High abundance fungi (RA $>1 \%$ ) were slightly higher in the non-rhizosphere environment, while medium abundance fungi increased in the rhizosphere environment. The extent of variation for low abundance fungi was substantially higher than that of high and medium abundance 
fungi. Therefore, fungi with different abundances have different responses to the pepper rhizosphere, among which the low abundance fungi were influenced the most. This is consistent with a previous report indicating that the change of soil environment has a greater impact on low abundance fungi than those of high abundance [35]. Studies have shown that microbiomes with different abundances exhibit different distribution patterns and functions [36]. In the warm and humid environment, the low abundance fungi increased [34]. This also verifies that the low abundance fungi were the main members responsible for the difference between the rhizosphere and non-rhizosphere soils in the special planting environment associated with plastic shed cultivation.

The OTU network relationship of the microbes also showed significant differences (Figure 3), with a positive correlation accounting for $70 \%$ within the rhizosphere and $81 \%$ within the non-rhizosphere environment. Accordingly, the main nodes of the network diagram were explored. The size of the node reflects the degree to which the fungi were involved in the interaction [37]. Generally, the nodes in the rhizosphere fungal network were greater than those in the bulk soil, leading to a balanced community network in the rhizosphere. Lasiosphaeriaceae (OTU70) and part 1 are the dominant members for the communities of the rhizosphere, while part 2 members dominated the fungal community network of the non-rhizosphere. Among them, part 1 members of the rhizosphere included Pseudeurotium, Scedosporium, Eurotiales, Phialocephala, Zopfiella, Pseudogymnoascus and others fungi, while the part 2 members of the non-rhizosphere included Waitea, Acremonium, Hypocreales, Fusarium, Chaetomiaceae, Cercophora. Therefore, it is interesting that the fungi involved in part 1 and part 2 were completely different but all classified as low abundance fungi, leading to the overall changes of the fungal community structure. The study explained that Lasiosphaeriaceae is associated with the available carbon sources, and it is also regarded as a potential disease suppressor in plants [38]. Although the abundance of Hypocreales was significantly enhanced under the rhizosphere environment, its node appeared in part 2 of the non-rhizosphere soil. This could be because the order Hypocreales, which includes the genera Purpureocillium, Metacorgyceps and Arthrobotrys regard as the largest source of biocontrol-related fungi and when the abundance of Hypocreales is high in the rhizosphere, its interaction with other fungi are weakened [39]. Thus, the rhizosphere environment can recruit certain fungal members, specifically those of benefit, to alter the fungal community [24].
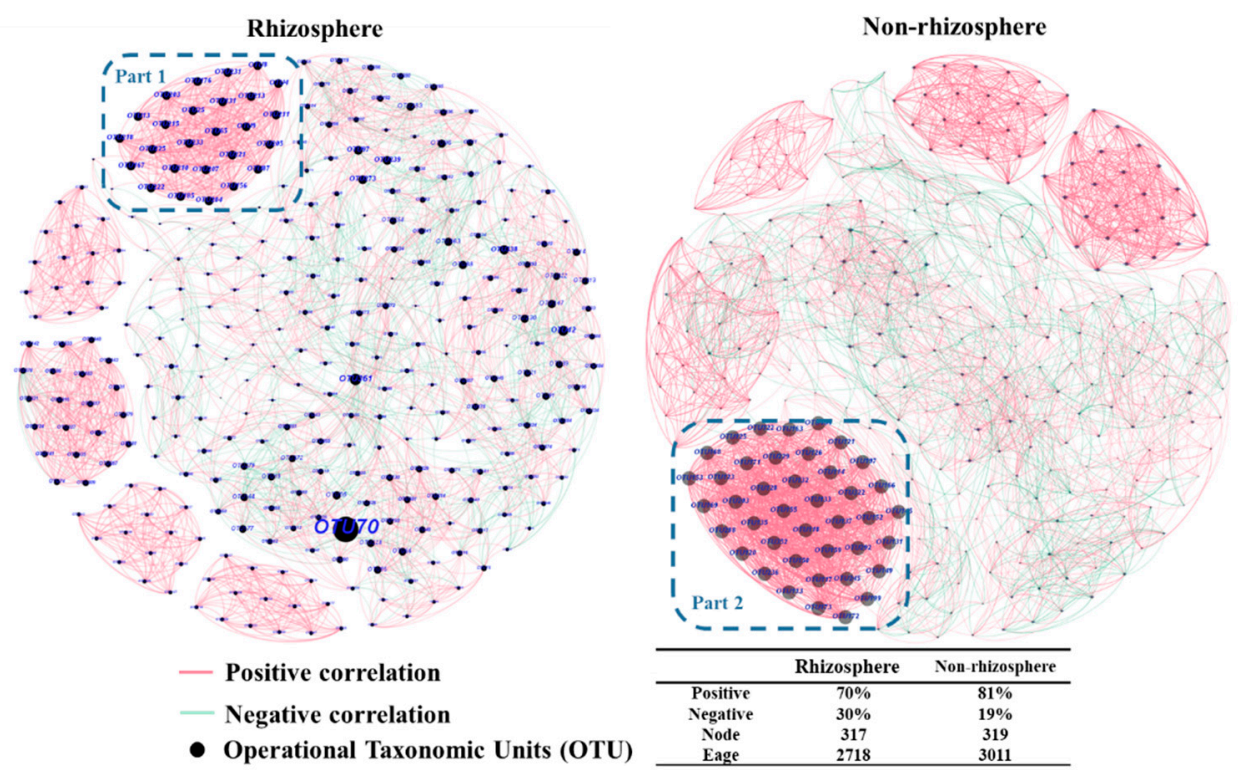

Figure 3. Co-occurrence network of the differential operational taxonomic units in soils.

\subsection{Shifts in the Fungal Functional Role in Soil}

Each soil environment had some specific functional roles with 41 and 31 unique function roles identified in the non-rhizosphere and rhizosphere environments, respectively. Therefore, the pepper 
rhizosphere not only changes the soil fungal community structure but also the functional roles of the fungal community.

Based on the trophic mode of fungal OTU, the fungal community functional roles could be divided into nine categories, including saprotroph, pathotroph, symbiotroph, saprotroph-symbiotroph, pathotroph-saprotroph-symbiotroph and other (Figure 4a). Saprotrophs dominated both the rhizosphere soil and non-rhizosphere soil, accounting for $54.66 \%$ and $44.12 \%$, respectively. Within the fungal community, the high fertilizer application and environmental temperature during plastic shed cultivation would be beneficial for the development of saprotroph functions [40]. The next most dominant tropic modes were the saprotroph-symbiotroph and pathotroph-saprotroph-symbiotroph transitional types. However, $36.75 \%$ of fungal community functional roles were not classified. Nguyen et al. showed that FUNGuild can provide the main information related to fungal functions, but improving the overall fungal functional information is still in progress [19].

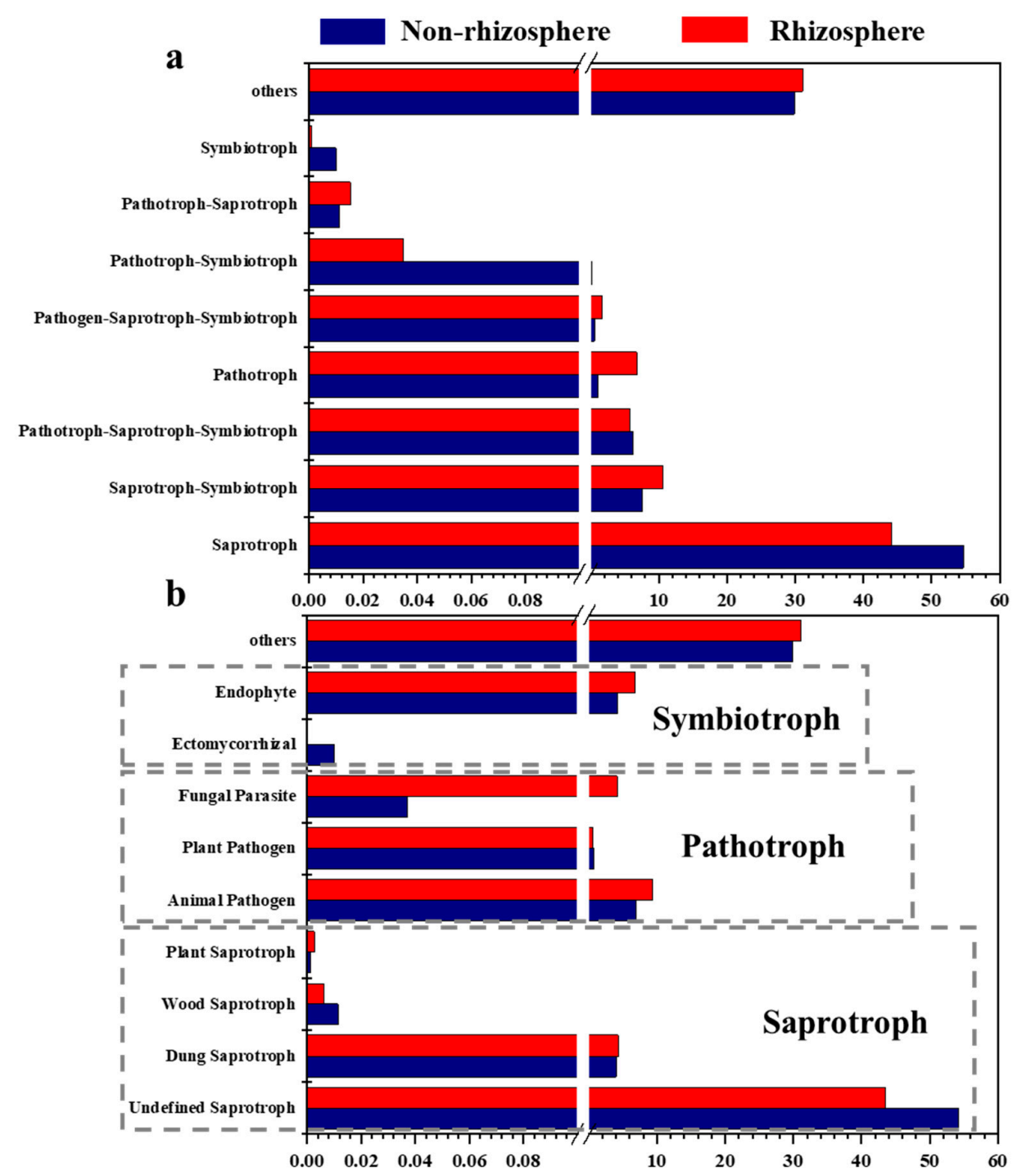

Figure 4. The abundance of different functional roles (a) and main guilds (b) assigned to the fungal community using FUNGuild.

The abundance of the pathtroph-symbiotroph, saprotroph, symbiotroph and pathotroph-saprotroph-symbiotroph trophic modes were higher in the non-rhizosphere soil than those in the rhizosphere soil, while the pathotroph, pathotroph-saprotroph, saprotroph-symbiotroph and other modes were lower in the non-rhizosphere environment than those in the rhizosphere environment 
(Figure 4a). Saprotroph is related to the role of fungi in decomposition. The abundance of saprotroph in rhizosphere soil was lower than that in non-rhizosphere soil in this study. Kong et al. also found that the non-rhizosphere condition enriched the relative abundance of saprotroph [37]. The increase of pathotrophic fungi in rhizosphere soil could be ascribed to the plant roots, although some pathogens attack the root system of crops, while others, such as some fungal parasites, could promote the healthy growth of crops [40]. Another study found that pathotrophic fungi could compete with other microbiomes in plant rhizosphere [9]. The relative abundance of fungal parasites and endophytes showed an obvious increase in the pepper rhizosphere soil under the plastic shed condition (Figure $4 \mathrm{~b}$ ). Within saprotroph, undefined saprotroph and wood saprotroph are more common in non-rhizosphere environments (Figure $4 \mathrm{~b}$ ), which is demonstrated by the higher nutrient utilization in this environment [41]. Meanwhile, the rhizosphere and non-rhizosphere soils contain many dung saprotroph, which is closely related to the application of organic fertilizer during plastic shed cultivation. Some studies have suggested that the changes of fungal functional roles were related to many factors, and plant species were also considered as important influencing factors [42,43]. Therefore, the difference between rhizosphere and non-rhizosphere fungal functional roles can be attributed to plant species and environmental factors.

\subsection{Co-Occurrence Network among the Fungal Community, Functional Roles and Soil Properties}

Soil properties drive the shifts in the soil fungal community structure and functional roles [44]. Furthermore, extensive use of plastic films could result in PAE contamination in the soils [5]. Therefore, to clarify their relationships, co-occurrence network analysis was performed between the soil properties, PAE, fungal functional roles and the significantly different fungal members (Figure 5). The co-occurrence network is composed of 32 nodes and 59 edges (Figure 5). The largest nodes in the network for fungal members, functional roles and soil properties represented Hypocreales, pathotroph functional roles and PAE, respectively. In the pepper rhizosphere, the members Hypocreales, Metacordyceps, unclassified_f_Lasiosphaeriaceae showed higher abundances than those in the non-rhizosphere and they positively correlated with PAE and pathotroph function. Conversely, the abundant fungal members Pezizomycetes, Pseudaleuria and Pezizales in the non-rhizosphere soil mainly showed negative correlations with PAE and pathotroph function.

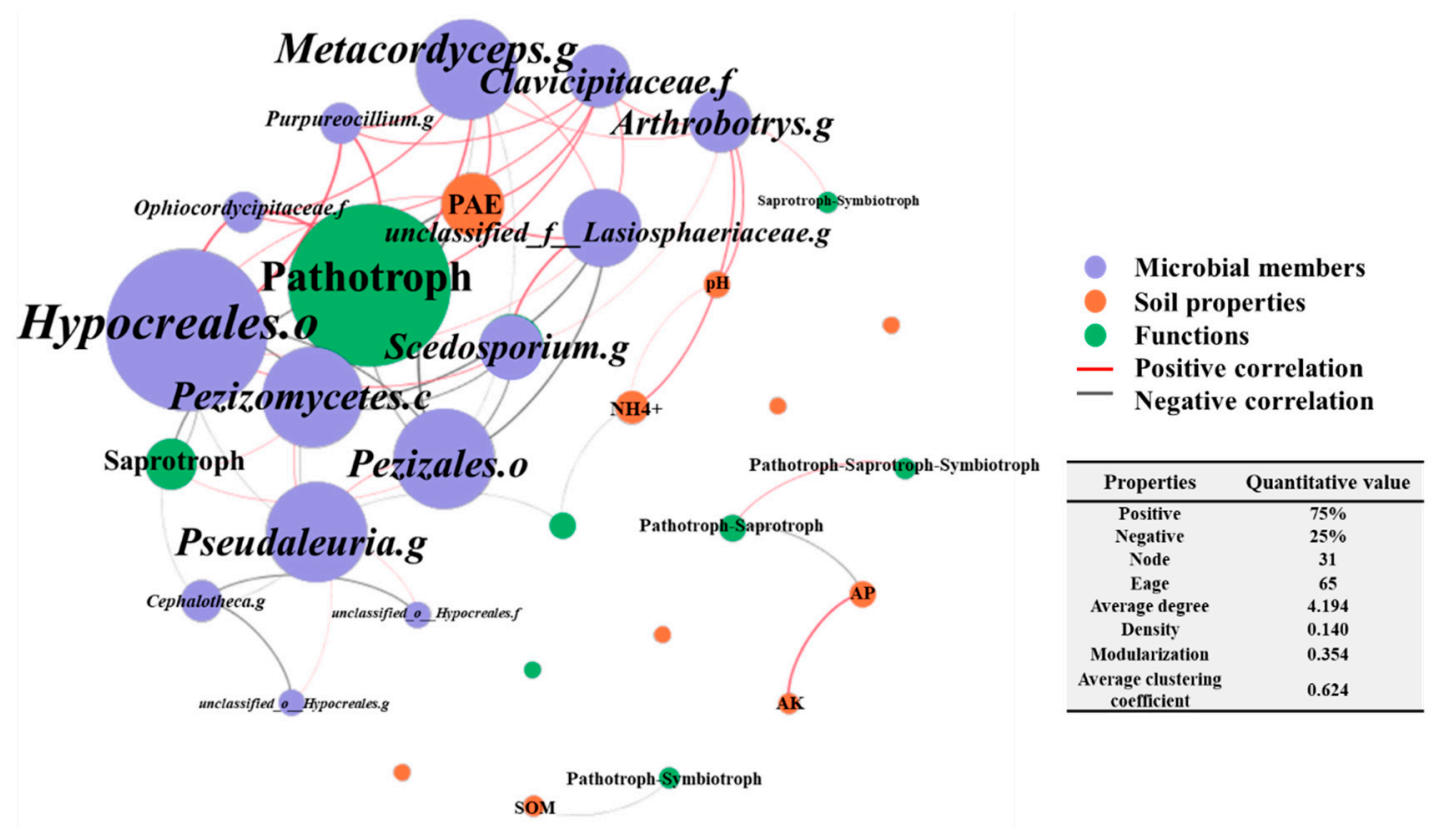

Figure 5. Co-occurrence network of the significantly different fungal members, predicted fungal functional roles and the soil physicochemical properties. 
The order Hypocreales, in the phylum Ascomycetes is considered to be the most abundant source of biocontrol-related fungi, which can inhibit insects, nematodes and other fungal species considered as plant pest populations [39]. Interestingly, Hypocreales showed positive correlations with both pathotroph function and PAE. Pathotroph function contains not only plant and animal pathogens, but also fungal parasites whose abundances were significantly greater in the rhizosphere than that in the bulk soils. Schmidt et al. reported that some fungal parasites could confer resistance to plant diseases and insect pests [40]. Therefore, the positive correlation between Hypocreales and pathotroph function may primarily be derived from the cooperation between Hypocreales and fungal parasites. The rhizosphere microbiomes are greatly affected by plant root exudates. Because of the large amount of organic and chemical fertilizers have been applied to soil under plastic shed cultivation, the differences in the soil properties between rhizosphere and non-rhizosphere were masked [32]. Therefore, common soil properties did not form central nodes in the network analysis (Figure 5). However, PAE, such as diethyl phthalate, di-isobutyl phthalate, di-n-butyl phthalate and di-n-octyl phthalate, have been reported as allelopathic substances of pepper root exudates [45]. It was found that the contents of phthalate, di-isobutyl phthalate, din-butyl phthalate and di-n-octyl phthalate in the pepper rhizosphere soil were greater than that those in bulk soil in our previous study [32]. Therefore, it could be inferred that the positive correlations between biocontrol fungi (Hypocreales) and PAE resulted in the positive release of PAE from the pepper root, which recruits certain fungal members. In a word, this case study indicate that the pepper roots recruit special biocontrol functional fungi and low abundance fungal taxa to construct a different and more balanced fungal community network in the rhizosphere, thereby benefitting growth under plastic shed cultivation [46,47].

\section{Conclusions}

During the intensive planting mode of plastic shed cultivation, low abundance fungi showed a sensitive response to the pepper rhizosphere, leading to a higher community diversity in the rhizosphere relative to the non-rhizosphere soil. Purpureocillium, Metacorgyceps and Arthrobotrys, as well as other unique fungi genera beneficial to plant growth, were recruited by the pepper root, thereby forming a differential network of the fungal community structure and functional roles. The rare members with low abundances of fungi deserved to be concerned for insight into the soil microbial ecology under plastic shed cultivation.

Author Contributions: Conceptualization, S.Y. and X.L.; Data curation, X.L.; Formal analysis, S.Y. and X.L.; Funding acquisition, Y.S.; Investigation, S.Y. and X.L.; Project administration, Y.S.; Supervision, Y.S.; Writing - original draft, S.Y. and X.L.; Writing-review \& editing, H.C., K.S., X.J. and Y.S. All authors have read and agreed to the published version of the manuscript.

Funding: This study was financially supported by the National Natural Science Foundation of China (41877032), Key Research Program of Frontier Sciences, Chinese Academy of Sciences (QYZDJ-SSW-DQC035), Opening Fund of National Engineering Laboratory for Site Remediation Technologies (NEL-SRT201904) and Agricultural Scientific and Technological Innovation Project of Shandong Academy of Agricultural Sciences (CXGC2016A06). And the APC was funded by (41877032).

Conflicts of Interest: The authors declare no conflict of interest.

\section{References}

1. Chang, J.; Wu, X.; Wang, Y.; Meyerson, L.A.; Gu, B.J.; Min, Y.; Xue, H.; Peng, C.H.; Ge, Y. Does growing vegetables in plastic greenhouses enhance regional ecosystem services beyond the food supply? Front. Ecol. Environ. 2013, 11, 43-49. [CrossRef]

2. Sun, J.T.; Pan, L.L.; Li, Z.H.; Zeng, Q.T.; Wang, L.W.; Zhu, L.Z. Comparison of greenhouse and open field cultivations across China: Soil characteristics, contamination and microbial diversity. Environ. Pollut. 2018, 243, 1509-1516. [CrossRef] [PubMed]

3. Gao, Y.Y.; Yang, S.; Li, X.X.; He, L.F.; Zhu, J.M.; Mu, W.; Liu, F. Residue determination of pyraclostrobin, picoxystrobin and its metabolite in pepper fruit via UPLC-MS/MS under open field conditions. Ecotox. Environ. Safe 2019, 182, e109445. [CrossRef] [PubMed] 
4. Wang, X.Z.; Liu, B.; Wu, G.; Sun, Y.X.; Guo, X.S.; Jin, Z.H.; Xu, W.N.; Zhao, Y.Z.; Zhang, F.S.; Zou, C.Q.; et al. Environmental costs and mitigation potential in plastic-greenhouse pepper production system in China: A life cycle assessment. Agric. Syst. 2018, 167, 186-194. [CrossRef]

5. Song, Y.; Xu, M.; Li, X.N.; Bian, Y.R.; Wang, F.; Yang, X.L.; Gu, C.G.; Jiang, X. Long-Term Plastic Greenhouse Cultivation Changes Soil Microbial Community Structures: A Case Study. J. Agric. Food. Chem. 2018, 66, 8941-8948. [CrossRef] [PubMed]

6. Hu, W.Y.; Zhang, Y.X.; Huang, B.; Teng, Y. Soil environmental quality in greenhouse vegetable production systems in eastern China: Current status and management strategies. Chemosphere 2017, 170, 183-195. [CrossRef]

7. Ning, Q.; Chen, L.; Jia, Z.J.; Zhang, C.Z.; Ma, D.H.; Li, F.; Zhang, J.B.; Li, D.M.; Han, X.R.; Cai, Z.J.; et al. Multiple long-term observations reveal a strategy for soil $\mathrm{pH}$-dependent fertilization and fungal communities in support of agricultural production. Agric. Ecosyst. Environ. 2020, 293, e106837. [CrossRef]

8. Van der Wal, A.; Geydan, T.D.; Kuyper, T.W.; de Boer, W. A thready affair: Linking fungal diversity and community dynamics to terrestrial decomposition processes. FEMS Microbiol. Rev. 2013, 37, 477-494. [CrossRef]

9. Martinez-Diz, M.D.; Andres-Sodupe, M.; Bujanda, R.; Diaz-Losada, E.; Eichmeier, A.; Gramaje, D. Soil-plant compartments affect fungal microbiome diversity and composition in grapevine. Fungal Ecol. 2019, 41, 234-244. [CrossRef]

10. Jangid, K.; Williams, M.A.; Franzluebbers, A.J.; Schmidt, T.M.; Coleman, D.C.; Whitman, W.B. Land-use history has a stronger impact on soil microbial community composition than aboveground vegetation and soil properties. Soil Biol. Biochem. 2011, 43, 2184-2193. [CrossRef]

11. Yao, Z.Y.; Xing, J.J.; Gu, H.P.; Wang, H.Z.; Wu, J.J.; Xu, J.M.; Brookes, P.C. Development of microbial community structure in vegetable-growing soils from open-field to plastic-greenhouse cultivation based on the PLFA analysis. J. Soils Sediments 2016, 16, 2041-2049. [CrossRef]

12. Compant, S.; Samad, A.; Faist, H.; Sessitsch, A. A review on the plant microbiome: Ecology, functions, and emerging trends in microbial application. J. Adv. Res. 2019, 19, 29-37. [CrossRef] [PubMed]

13. Majid, M.U.; Awan, M.F.; Fatima, K.; Tahir, M.S.; Ali, Q.; Rashid, B.; Rao, A.Q.; Nasir, I.A.; Husnain, T. Phytophthora capsici on chilli pepper (Capsicum annuum L.) and its management through genetic and bio-control: A review. Zemdirb. Agric. 2016, 103, 419-430. [CrossRef]

14. Sergaki, C.; Lagunas, B.; Lidbury, I.; Gifford, M.L.; Schafer, P. Challenges and approaches in microbiome research: From fundamental to applied. Front. Plant Sci. 2018, 9. [CrossRef]

15. Li, X.G.; Ding, C.F.; Hua, K.; Zhang, T.L.; Zhang, Y.N.; Zhao, L.; Yang, Y.R.; Liu, J.G.; Wang, X.X. Soil sickness of peanuts is attributable to modifications in soil microbes induced by peanut root exudates rather than to direct allelopathy. Soil Biol. Biochem. 2014, 78, 149-159. [CrossRef]

16. Li, S.L.; Xu, C.; Wang, J.; Guo, B.; Yang, L.; Chen, J.N.; Ding, W. Cinnamic, myristic and fumaric acids in tobacco root exudates induce the infection of plants by Ralstonia solanacearum. Plant Soil 2017, 412, 381-395. [CrossRef]

17. Li, Z.G.; Zu, C.; Wang, C.; Yang, J.F.; Yu, H.; Wu, H.S. Different responses of rhizosphere and non-rhizosphere soil microbial communities to consecutive Piper nigrum L. monoculture. Sci. Rep. 2016, 6. [CrossRef]

18. Wang, Y.J.; Liu, L.; Yang, J.F.; Duan, Y.M.; Luo, Y.; Taherzadeh, M.J.; Li, Y.F.; Li, H.K.; Awasthi, M.K.; Zhao, Z.Y. The diversity of microbial community and function varied in response to different agricultural residues composting. Sci. Total Environ. 2020, 715, e136983. [CrossRef]

19. Nguyen, N.H.; Song, Z.W.; Bates, S.T.; Branco, S.; Tedersoo, L.; Menke, J.; Schilling, J.S.; Kennedy, P.G. FUNGuild: An open annotation tool for parsing fungal community datasets by ecological guild. Fungal Ecol. 2016, 20, 241-248. [CrossRef]

20. Feng, H.J.; Wang, S.Y.; Gao, Z.D.; Wang, Z.K.; Ren, X.Q.; Hu, S.W.; Pan, H. Effect of land use on the composition of bacterial and fungal communities in saline-sodic soils. Land Degrad. Dev. 2019, 30, 1851-1860. [CrossRef]

21. Lian, T.X.; Mu, Y.H.; Jin, J.; Ma, Q.B.; Cheng, Y.B.; Cai, Z.D.; Nian, H. Impact of intercropping on the coupling between soil microbial community structure, activity, and nutrient-use efficiencies. Peer J. 2019, 7, e6412. [CrossRef] [PubMed] 
22. Skaltsas, D.N.; Badotti, F.; Vaz, A.B.M.; da Silva, F.F.; Gazis, R.; Wurdack, K.; Castlebury, L.; Goes-Neto, A.; Chaverri, P. Exploration of stem endophytic communities revealed developmental stage as one of the drivers of fungal endophytic community assemblages in two Amazonian hardwood genera. Sci. Rep. 2019, 9. [CrossRef] [PubMed]

23. Nie, S.A.; Lei, X.M.; Zhao, L.X.; Brookes, P.C.; Wang, F.; Chen, C.R.; Yang, W.H.; Xing, S.H. Fungal communities and functions response to long-term fertilization in paddy soils. Appl. Soil Ecol. 2018, 130, 251-258. [CrossRef]

24. Berendsen, R.L.; Pieterse, C.M.J.; Bakker, P.A.H.M. The rhizosphere microbiome and plant health. Trends Plant Sci. 2012, 17, 478-486. [CrossRef]

25. Liu, H.; Pan, F.J.; Han, X.Z.; Song, F.B.; Zhang, Z.M.; Yan, J.; Xu, Y.L. A comprehensive analysis of the response of the fungal community structure to long-term continuous cropping in three typical upland crops. J. Integr. Agric. 2020, 19, 866-880. [CrossRef]

26. Dahlin, P.; Eder, R.; Consoli, E.; Krauss, J.; Kiewnick, S. Integrated control of Meloidogyne incognita in tomatoes using fluopyram and Purpureocillium lilacinum strain 251. Crop Prot. 2019, 124, e104874. [CrossRef]

27. Moreno-Salazar, R.; Sanchez-Garcia, I.; Chan-Cupul, W.; Ruiz-Sanchez, E.; Hernandez-Ortega, H.A.; Pineda-Lucatero, J.; Figueroa-Chavez, D. Plant growth, foliar nutritional content and fruit yield of Capsicum chinense biofertilized with Purpureocillium lilacinum under greenhouse conditions. Sci. Hortic. 2020, 261, e108950. [CrossRef]

28. Strom, N.; Hu, W.M.; Haarith, D.; Chen, S.Y.; Bushley, K. Corn and soybean host root endophytic fungi with toxicity toward the soybean cyst nematode. Phytopathology 2020, 110, 603-614. [CrossRef]

29. Nimnoi, P.; Ruanpanun, P. Suppression of root-knot nematode and plant growth promotion of chili (Capsicum flutescens L.) using co-inoculation of Streptomyces spp. Biol. Control 2020, 145, e104244. [CrossRef]

30. Gine, A.; Carrasquilla, M.; Martinez-Alonso, M.; Gaju, N.; Sorribas, F.J. Characterization of soil suppressiveness to root-knot nematodes in organic horticulture in plastic greenhouse. Front. Plant Sci. 2016, 7, e00164. [CrossRef]

31. Mouhajir, A.; Poirier, W.; Angebault, C.; Rahal, E.; Bouabid, R.; Bougnoux, M.E.; Kobi, A.; Zouhair, R.; Bouchara, J.P.; Giraud, S. Scedosporium species in soils from various biomes in Northwestern Morocco. PLoS ONE 2020, 15, e0228897. [CrossRef] [PubMed]

32. Song, Y.; Li, X.; Yao, S.; Yang, X.; Jiang, X. Correlations between soil metabolomics and bacterial community structures in the pepper rhizosphere under plastic greenhouse cultivation. Sci. Total Environ. 2020, 728, 138439. [CrossRef] [PubMed]

33. Xiang, X.J.; Liu, J.; Zhang, J.; Li, D.M.; Xu, C.X.; Kuzyakov, Y. Divergence in fungal abundance and community structure between soils under long-term mineral and organic fertilization. Soil Tillage Res. 2020, 196, 104491. [CrossRef]

34. Jiao, S.; Lu, Y. Abundant fungi adapt to broader environmental gradients than rare fungi in agricultural fields. Glob. Chang. Biol. 2020. [CrossRef]

35. Song, Y.; Li, X.N.; Xu, M.; Jiao, W.; Bian, Y.R.; Yang, X.L.; Gu, C.G.; Wang, F.; Jiang, X. Does biochar induce similar successions of microbial community structures among different soils? Bull. Environ. Contam. Toxicol. 2019, 103, 642-650. [CrossRef]

36. Pedros-Alio, C. The rare bacterial biosphere. Annu. Rev. Mar. Sci. 2012, 4, 449-466. [CrossRef]

37. Kong, X.; Jin, D.C.; Wang, X.X.; Zhang, F.S.; Duan, G.L.; Liu, H.J.; Jia, M.H.; Deng, Y. Dibutyl phthalate contamination remolded the fungal community in agro-environmental system. Chemosphere 2019, 215, 189-198. [CrossRef]

38. Liu, L.L.; Huang, X.Q.; Zhao, J.; Zhang, J.B.; Cai, Z.C. Characterizing the key agents in a disease-suppressed soil managed by reductive soil disinfestation. Appl. Environ. Microbiol. 2019, 85, 15. [CrossRef]

39. Kepler, R.M.; Maul, J.E.; Rehner, S.A. Managing the plant microbiome for biocontrol fungi: Examples from Hypocreales. Curr. Opin. Microbiol. 2017, 37, 48-53. [CrossRef]

40. Schmidt, R.; Mitchell, J.; Scow, K. Cover cropping and no-till increase diversity and symbiotroph: Saprotroph ratios of soil fungal communities. Soil Biol. Biochem. 2019, 129, 99-109. [CrossRef]

41. Chen, W.Q.; Wang, J.Y.; Meng, Z.X.; Xu, R.; Chen, J.; Zhang, Y.J.; Hu, T.M. Fertility-related interplay between fungal guilds underlies plant richness-productivity relationships in natural grasslands. New Phytol. 2020, 226, 1129-1143. [CrossRef] [PubMed] 
42. Kolarikova, Z.; Kohout, P.; Kruger, C.; Janouskova, M.; Mrnka, L.; Rydlova, J. Root-associated fungal communities along a primary succession on a mine spoil: Distinct ecological guilds assemble differently. Soil Biol. Biochem. 2017, 113, 143-152. [CrossRef]

43. Tedersoo, L.; Bahram, M.; Cajthaml, T.; Polme, S.; Hiiesalu, I.; Anslan, S.; Harend, H.; Buegger, F.; Pritsch, K.; Koricheva, J.; et al. Tree diversity and species identity effects on soil fungi, protists and animals are context dependent. ISME J. 2016, 10, 346-362. [CrossRef] [PubMed]

44. Fierer, N. Embracing the unknown: Disentangling the complexities of the soil microbiome. Nat. Rev. Microbiol. 2017, 15, 579-590. [CrossRef]

45. Sun, H.Y.; Wang, Y. Potential allelopathic effects of allelochemicals in aqueous extracts of leaves and root exudates of Capsicum annuum on vegetable crops. Allelopath. J. 2015, 35, 11-22.

46. Floc'h, J.B.; Hamel, C.; Harker, K.N.; St-Arnaud, M. Fungal communities of the canola rhizosphere: Keystone species and substantial between-year variation of the rhizosphere microbiome. Microb. Ecol. 2020. [CrossRef]

47. Kokalis-Burelle, N.; McSorley, R.; Wang, K.H.; Saha, S.K.; McGovern, R.J. Rhizosphere microorganisms affected by soil solarization and cover cropping in Capsicum annuum and Phaseolus lunatus agroecosystems. Appl. Soil Ecol. 2017, 119, 64-71. [CrossRef]

Publisher's Note: MDPI stays neutral with regard to jurisdictional claims in published maps and institutional affiliations.

(C) 2020 by the authors. Licensee MDPI, Basel, Switzerland. This article is an open access article distributed under the terms and conditions of the Creative Commons Attribution (CC BY) license (http://creativecommons.org/licenses/by/4.0/). 